\title{
Migration des macro et oligo-éléments dans un fromage à pâte molle de type Camembert
}

\author{
Y. LE GRAET et G. BRULÉ \\ INRA, Laboratoire de Recherches de Technologie laitière \\ 65, rue de Saint-Brieuc, 35042 Rennes Cedex, France
}

\section{Résumé}

Au cours de l'affinage des fromages à pâte molle de type Camembert, certains minéraux migrent de l'intérieur vers l'extérieur de façon irréversible. Ainsi les teneurs en calcium et magnésium de la croûte en fin d'affinage sont respectivement de $16,7 \mathrm{~g} /$ $\mathrm{kg}$ et de $521,9 \mathrm{mg} / \mathrm{kg}$ alors que celles du centre sont de $0,92 \mathrm{~g} / \mathrm{kg}$ et de $72,5 \mathrm{mg} / \mathrm{kg}$. Ces minéraux se trouvent associés à l'ion phosphate et forment un sel insoluble qui précipite à la surface sous l'action du gradient de $\mathrm{pH}$.

La migration du potassium est réversible : $1,25 \mathrm{~g} / \mathrm{kg}$ à 5 jours, $3,37 \mathrm{~g} / \mathrm{kg}$ à 9 jours et à nouveau $1,24 \mathrm{~g} / \mathrm{kg}$ à 19 jours dans la croûte; cette migration se fait sous l'action de la flore de surface et non du gradient de $\mathrm{pH}$.

Après 19 jours d'affinage, la teneur en sodium de la croûte s'élève à $3,33 \mathrm{~g} / \mathrm{kg}$, et celle du centre du fromage à $5,37 \mathrm{~g} / \mathrm{kg}$, alors qu'à 5 jours la répartition est homogène.

Le zinc présente, comme le potassium, une migration réversible mais moins prononcée dans la $2^{\mathrm{C}}$ phase. A 9 jours la teneur de la croûte est maximum et atteint $89,6 \mathrm{mg} / \mathrm{kg}$; à 19 jours elle est de $57,6 \mathrm{mg} / \mathrm{kg}$. Les teneurs dans le centre pour la même période sont de 18,3 et $20,4 \mathrm{mg} / \mathrm{kg}$.

Le fer migre sous l'influence du gradient de $\mathrm{pH}$; sa teneur dans la croûte double durant la période d'affinage et atteint $2,67 \mathrm{mg} / \mathrm{kg}$ à 19 jours.

Le cuivre fortement fixé aux protéines ne migre pas et la teneur moyenne est de $0,50 \mathrm{mg} / \mathrm{kg}$.

Mots clés : Affinage - Camembert - Minéraux - Calcium - Magnésium - Sodium Potassium - Zinc - Fer - Cuivre - Ammoniac.

\section{Summary}

\section{Mineral migration in soft cheeses during ripening}

During ripening of soft cheese, some of mineral salts move from inside to outside. Amounts of calcium and magnesium in the external rind at the end of ripening are respectively $16.7 \mathrm{~g} / \mathrm{kg}$ and $521.9 \mathrm{mg} / \mathrm{kg}$ against $0.92 \mathrm{~g} / \mathrm{kg}$ and $72.7 \mathrm{mg} / \mathrm{kg}$ in the central part. These minerals are associated with phosphate ion and precipitate under $\mathrm{pH}$ gradient effect.

The potassium migration is reversible; the rind content is $1.25 \mathrm{~g} / \mathrm{kg}$ at 5 days, $3.37 \mathrm{~g} / \mathrm{kg}$ at 9 days and $1.24 \mathrm{~g} / \mathrm{kg}$ at 19 days; this transfer is only related to the growth of $P$. Camemberti. $\mathrm{pH}$ gradient has no effect. 

part.

The amount of sodium in the cheese rind is $3.33 \mathrm{~g} / \mathrm{kg}$ and $5.37 \mathrm{~g} / \mathrm{kg}$ in the central

Zinc, as potassium has a reversible migration but the centripetal one is less important ; amount of zinc is $89.6 \mathrm{mg} / \mathrm{kg}$ at 9 days, $57.6 \mathrm{mg} / \mathrm{kg}$ at 19 days in the rind and during the same time amounts are respectively 18.3 and $20.4 \mathrm{mg}$ in the central part.

Iron migration is low under $\mathrm{pH}$ gradient ; its amount increased twofold during ripening to reach $2.67 \mathrm{mg} / \mathrm{kg}$ at 19 days.

Because of a strong proteins-copper binding there is no migration of the cation and its average amount is $0.5 \mathrm{mg} / \mathrm{kg}$ of cheese.

Key words : Ripening - Camembert - Minerals - Calcium - Magnesium - Sodium Potassium - Zinc - Iron - Copper.

\section{Introduction}

L'étude de l'évolution de la répartition du calcium et du phosphate, dans les fromages à pâte molle de type Camembert, au cours de l'affinage, a montré le rôle prépondérant du $\mathrm{pH}$ dans le phénomène de migration de ces éléments (LE GRAet et al., 1983). Les transferts de l'ensemble des macro et oligo-éléments étudiés au cours de l'affinage des fromages de type pâte cuite (Le Graet et al., 1986) ont montré qu'il existait des besoins spécifiques de la flore de surface et confirmé l'influence du $\mathrm{pH}$; ces besoins ont fait l'objet de divers travaux dont ceux notamment de SaAKYan (1982) et Hofi et al. (1970).

Il nous a paru alors intéressant de réaliser une étude complète sur l'évolution de la répartition de l'ensemble des macro $(\mathrm{Ca}, \mathrm{Mg}, \mathrm{K}, \mathrm{Na}$ ) et oligo-éléments $(\mathrm{Zn}, \mathrm{Fe}, \mathrm{Cu})$ au cours de l'affinage de fromage de type pâte molle, compte tenu d'une part, de l'abondance du Penicillium et, d'autre part, de l'important gradient de $\mathrm{pH}$, pour apprécier le rôle spécifique de chacun d'eux. Pour atteindre cet objectif nous avons donc comparé des fromages affinés dans les conditions habituelles à ceux affinés en atmosphère non modifiée ou ammoniacale mais en présence d'un inhibiteur fongique.

\section{Matériel et méthodes}

\section{A. Fromages}

Les fromages à pâte molle de type Camembert étaient fabriqués à partir d'un lait pasteurisé $72{ }^{\circ} \mathrm{C}-15 \mathrm{~s}$ et avaient un rapport gras $/ \mathrm{sec}$ de $45 \%$. Ils nous étaient fournis après salage par la Laiterie TriBalLat (35530 Noyal-sur-Vilaine).

\section{B. Affinage}

Après salage une première série de fromages était divisée en 2 lots : l'un recevait une pulvérisation d'une suspension de spores de $P$. camemberti, l'autre était traité à l'aide d'une solution antifongique de pimaricine à $0.5 \%$ afin 
d'éviter tout développement de $P$. camemberti; cette série était affinée en atmosphère non modifiée $\left(12{ }^{\circ} \mathrm{C}\right.$, hygrométrie $\left.95 \%\right)$.

La $2^{\mathrm{e}}$ série de fromages, d'une autre fabrication, recevait une pulvérisation de pimaricine dans les mêmes conditions que la précédente, mais était affinée en atmosphère ammoniacale. L'enceinte d'affinage d'un volume de $0,75 \mathrm{~m}^{3}$ était ventilée ; la diffusion du gaz $\mathrm{NH}_{3}$ se faisait à partir d'un bécher d'un volume d'un litre contenant 0,51 d'une solution d'ammoniaque maintenue à $\mathrm{pH}$ constant $(9,5)$ à l'aide d'un $\mathrm{pH}$-Stat Mettler connecté à une réserve d'ammoniaque $0,3 \mathrm{~N}$.

Par ailleurs, les fromages étaient posés sur un store en plastique préalablement trempé dans une solution $\mathrm{HCl} 5 \mathrm{~N}$, puis rincé à l'eau bidistillée pour éliminer les contaminations en fer; de plus l'utilisation du store, meilleur support que les claies, en atmosphère ammoniacale, améliorait la « tenue » du fromage, ce qui a permis d'obtenir un gradient de $\mathrm{pH}$ comparable à celui d'un fromage traditionnel.

Les résultats présentés ne concernent qu'une expérimentation représentative des 8 réalisées au total, et dont la moyenne n'a pu être présentée compte tenu, d'une part, d'une évolution de l'affinage qui n'est pas rigoureusement identique.

\section{Découpe des fromages pour analyse}

Chaque fromage était découpé en 3 parties : la croûte, la partie souscroûte et le centre. Le détail de la découpe est présenté dans une précédente publication (LE GRAET et al., 1983).

\section{Déterminations analytiques}

\section{Cendres}

Elles sont obtenues par calcination d'environ $12 \mathrm{~g}$ de fromage ou de croûte à $530^{\circ} \mathrm{C}$ pendant 3 heures.

\section{Teneurs en minéraux}

Les déterminations des teneurs en calcium, magnésium, sodium, potassium, fer, zinc et cuivre étaient réalisées par spectrométrie d'absorption atomique sur un appareil VARIAN AA 1275 à partir des cendres obtenues sur les fromages et reprises dans $50 \mathrm{ml}$ d'acide chlorhydrique $0,1 \mathrm{~N}$. A partir de cette solution les dilutions étaient réalisées en fonction de la teneur en chacun des éléments.

Le dosage du phosphore total était effectué à partir de $5 \mathrm{ml}$ de la solution précédente, selon la méthode préconisée par la Fédération Internationale de Laiterie (norme 33).

\section{Teneurs en matière sèche}

Elle était déterminée par dessication à l'étuve à $102-105^{\circ} \mathrm{C}$ pendant $7 \mathrm{~h}$. 
4. Mesure du pH

Les déterminations de $\mathrm{pH}$ étaient effectuées avec un appareil PORTATEST 653 à 0,02 unité $\mathrm{pH}$ près.

\section{Résultats}

A. Caractéristiques physico-chimiques des fromages

1. $p H$

Les variations de $\mathrm{pH}$ des fromages sont représentées sur la fig. 1. Dans le cas du fromage couvert de penicillium, le pH évolue de 4,70 à 7,12 dans la croûte entre $\mathbf{J} 0$ ( $\mathbf{J} 0$ correspondant au fromage après salage) et $\mathbf{J}+19$; dans la partie sous-croûte, il s'élève de 4,62 à 6,82 de façon linéaire à partir du $5^{\mathrm{e}}$ jour. Dans le centre cette évolution n'est perceptible que le $9^{\mathrm{e}}$ jour ; il varie alors de 4,55 à 5,06 à 19 jours.
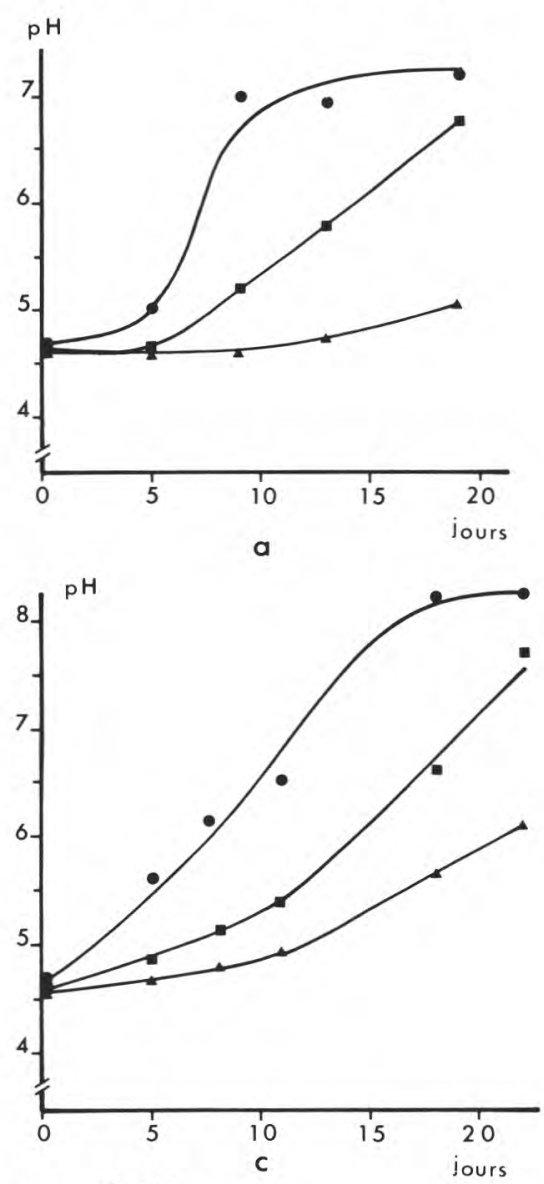

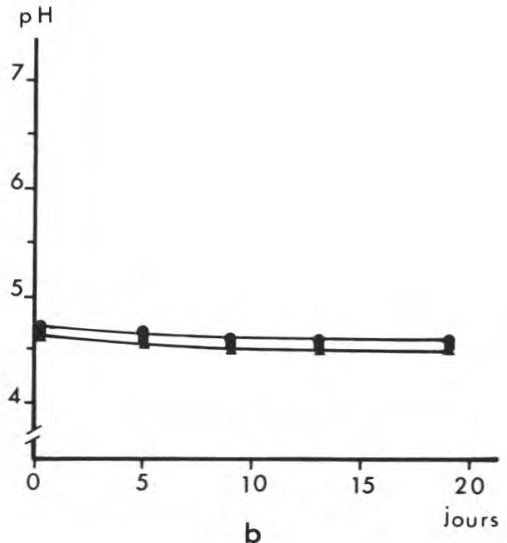

FIG. 1

Evolution du pH au cours de l'affinage.

$a$ : fromages ensemencés en $\mathrm{P}$. camemberti (atmosphère non modifiée).

$b$ : fromages traités à la pimaricine (atmosphère non modifiée).

$c$ : fromages traités à la pimaricine et placés en atmosphère d'ammoniac.

Evolution of $\mathrm{pH}$ during ripening.

$a$ : P. camemberti - Normal conditions.

$b$ : Pimaricin - Normal conditions.

$c$ : Pimaricin - $\mathrm{NH}_{3}$ conditions.

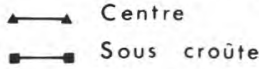

$\longrightarrow$ Croûte 
En présence de pimaricine, aucune variation de $\mathrm{pH}$ n'est décelée durant la période d'essai.

Sans développement de penicillium, mais en atmosphère ammoniacale, le $\mathrm{pH}$ de la croûte s'élève de 4,70 à $\mathrm{J} 0$ à 8,33 à $\mathrm{J}+22$. Dans la partie souscroûte, l'évolution est d'abord lente : 4,66 à $\mathrm{J} 0$ et 5,42 à $\mathrm{J}+11$ puis s'accélère pour atteindre 7,72 à 22 jours. Le $\mathrm{pH}$ centre évolue dans le même sens : 4,62 à $\mathbf{J} 0,4,95$ à $\mathrm{J}+11$ et 6,20 à 22 jours.

\section{Extrait sec}

En présence de penicillium l'extrait sec de la croûte diminue constamment de $49,7 \%$ à $\mathrm{J} 0$ à $34,9 \%$ à 19 jours (fig. 2 ). Il n'en est pas de même dans la partie sous-croûte et le centre où les extraits secs augmentent de façon parallèle pendant les 10 premiers jours pour devenir constants ensuite ; les teneurs sont respectivement 39,6 et $38,0 \%$ à $\mathbf{J} 0$ et 44,9 et 43,3 à 19 jours.
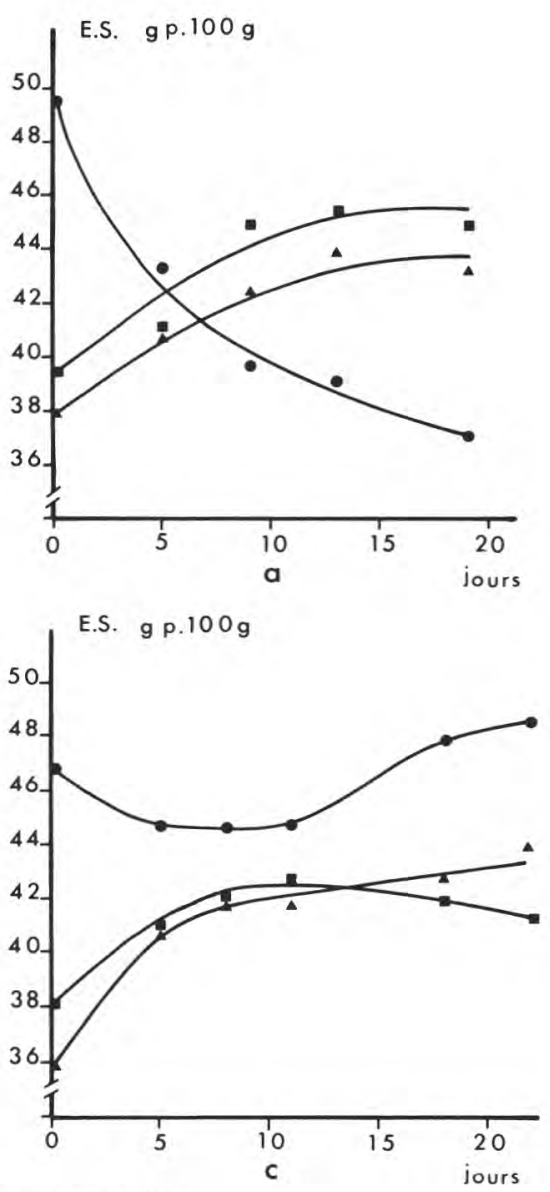

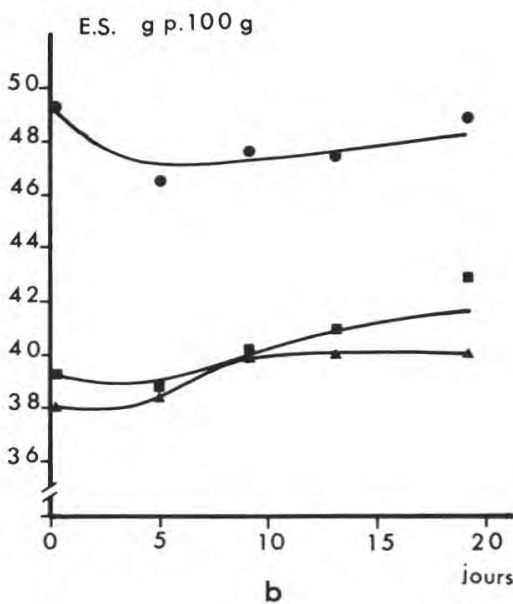

FIG. 2

Evolution de l'extrait sec au cours de l'affinage.

$a$ : fromages ensemencés en $\mathrm{P}$. camemberti (atmosphère non modifiée).

$b$ : fromages traités à la pimaricine (atmosphère non modifiée).

$c$ : fromages traités à la pimaricine et placés en atmosphère d'ammoniac.

Evolution of dry matter during ripening.

$a:$ P. camemberti - Normal conditions.

$b$ : Pimaricin - Normal conditions.

c: Pimaricin $-\mathrm{NH}_{3}$ conditions.

$\longrightarrow$ Croûte

Sous croûte

Centre 
Les fromages ayant reçu une pulvérisation de pimaricine et placés en atmosphère non modifiée ne subissent pas de variations importantes d'extrait sec ; dans la croûte il évolue de 49,7 à $46,2 \%$ de $\mathrm{J} 0$ à 5 jours et remonte à $49,0 \%$ à 19 jours. Durant la même période, l'extrait sec de la partie souscroûte et du centre augmente peu, respectivement de 39,6 et $38,0 \%$ à 43,0 et $40,0 \%$.

En atmosphère ammoniacale, l'extrait sec de la croûte baisse de $46,7 \%$ à $\mathrm{J} 0$ à $44,6 \%$ à 11 jours puis remonte à $48,6 \%$ à 22 jours. Dans la partie souscroûte il augmente entre $\mathbf{J} 0$ et $\mathbf{J}+11$ de $37,9 \%$ à $42,8 \%$, puis diminue lentement à $41,4 \%$ à 22 jours. Par contre, dans le centre l'extrait sec augmente rapidement : $35,7 \%$ à $\mathrm{J} 0,42,0 \%$ à 8 jours et $44,1 \%$ à 22 jours.

\section{B. Répartition des macro-éléments au cours de l'affinage}

\section{Evolution des teneurs en calcium}

Les fromages ensemencés en penicillium montrent que la teneur en calcium de la croûte augmente dans de fortes proportions entre 5 et 9 jours, de 3,25 à $15,39 \mathrm{~g} / \mathrm{kg}$ puis $16,69 \mathrm{~g} / \mathrm{kg}$ à 19 jours (fig. 3). Parallélement, la teneur en calcium de la partie sous-croûte diminue dans une première phase plus rapidement que celle du centre où les valeurs sont respectivement de 1,35 et $1,96 \mathrm{~g} / \mathrm{kg}$ à 9 jours, 1,00 et $0,92 \mathrm{~g} / \mathrm{kg}$ à 19 jours alors qu'à 5 jours elles sont semblables et s'élèvent à $3,03 \mathrm{~g} / \mathrm{kg}$.

En présence de pimaricine les teneurs identiques dans les 3 parties sont de $2,90 \mathrm{~g} / \mathrm{kg}$ à 5 jours et de $3,30 \mathrm{~g} / \mathrm{kg}$ environ à 19 jours.

En atmosphère ammoniacale on note dès 5 jours une augmentation de la teneur en calcium de la croûte et une diminution simultanée dans les 2 autres parties. A 5 jours, les teneurs en calcium de la croûte, partie sous-croûte et du centre sont de $5,71,2,41$ et $2,61 \mathrm{~g} / \mathrm{kg}$ alors qu'à 18 jours elles sont de 15,48 , 1,35 et $1,23 \mathrm{~g} / \mathrm{kg}$. 

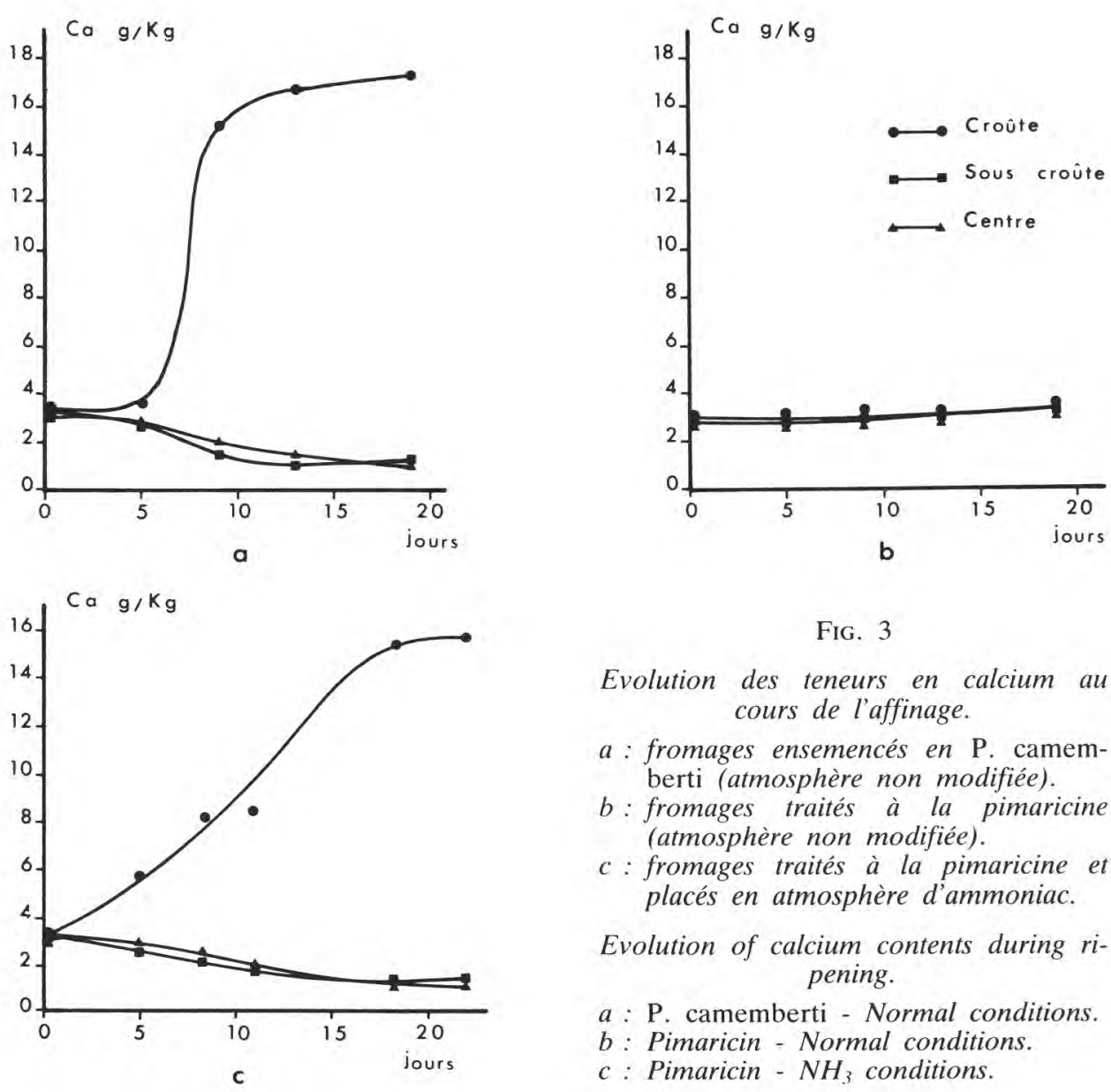

FIG. 3

Evolution des teneurs en calcium au cours de l'affinage.

$a$ : fromages ensemencés en $\mathrm{P}$. camemberti (atmosphère non modifiée).

$b$ : fromages traités à la pimaricine (atmosphère non modifiée).

$c$ : fromages traités à la pimaricine et placés en atmosphère d'ammoniac.

Evolution of calcium contents during ripening.

$a$ : P. camemberti - Normal conditions.

$b$ : Pimaricin - Normal conditions.

c: Pimaricin - $\mathrm{NH}_{3}$ conditions.

\section{Evolution des teneurs en magnésium}

Les fromages ensemencés en penicillium et affinés en atmosphère non modifiée présentent une courbe d'évolution des teneurs en magnésium identique à celle du calcium (fig. 4). Les quantités de magnésium contenues dans le centre, la partie sous-croûte et la croûte sont d'environ $137,0 \mathrm{mg} / \mathrm{kg}$ à 5 jours et respectivement $72,5,105,0$ et $521,9 \mathrm{mg} / \mathrm{kg}$ à 19 jours.

En présence de pimaricine, il n'existe aucune différence significative entre les 3 parties du fromage et les teneurs se situent aux environs de $140 \mathrm{mg} / \mathrm{kg}$ à 19 jours.

En atmosphère ammoniacale, dès le $5^{\mathrm{e}}$ jour, la teneur de la croûte est plus élevée que celle du reste du fromage. Les valeurs dans la croûte, partie souscroûte et le centre sont de $170,9,135,0$ et $132,8 \mathrm{mg} / \mathrm{kg}$ à 5 jours, de 501,8 , 72,5 et $70,1 \mathrm{mg} / \mathrm{kg}$ à 18 jours. 

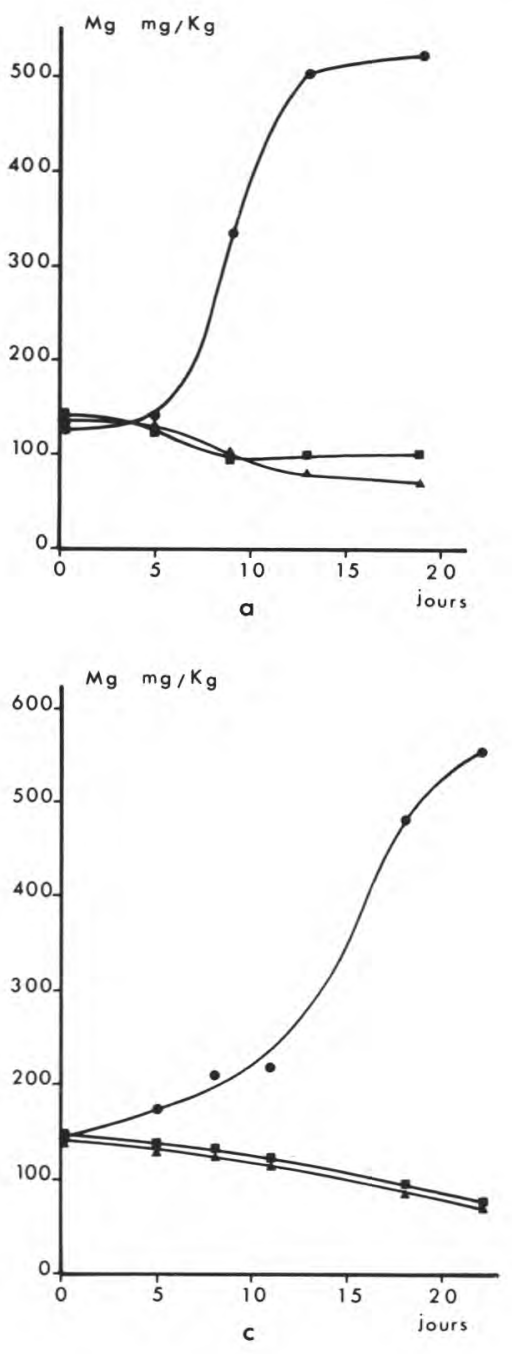

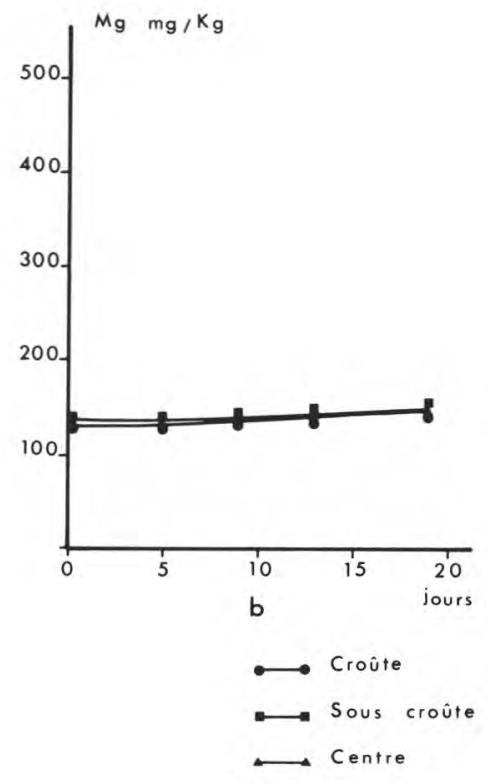

FIG. 4

Evolution des teneurs en magnésium au cours de l'affinage.

$a$ : fromages ensemencés en $\mathrm{P}$. camemberti (atmosphère non modifiée).

$b$ : fromages traités à la pimaricine (atmosphère non modifiée).

$c$ : fromages traités à la pimaricine et placés en atmosphère d'ammoniac.

Evolution of magnesium contents during ripening.

$a$ : P. camemberti - Normal conditions.

$b$ : Pimaricin - Normal conditions.

c : Pimaricin - $\mathrm{NH}_{3}$ conditions.

\section{Evolution des teneurs en sodium}

Après salage la teneur en sodium de la croûte est évidemment beaucoup plus élevée que celle du reste du fromage : $11,59 \mathrm{~g} / \mathrm{kg}$ dans cette dernière, $5,81 \mathrm{~g} / \mathrm{kg}$ dans la partie sous-croûte et $1,11 \mathrm{~g} / \mathrm{kg}$ dans le centre (fig. 5). A 5 jours, la répartition s'homogénéise et les valeurs sont comprises entre 5,21 et $5,65 \mathrm{~g} / \mathrm{kg}$. A 19 jours, il n'en est plus de même puisque la teneur de la croûte a nettement diminué pour atteindre $3,33 \mathrm{~g} / \mathrm{kg}$ alors que dans les parties souscroûte et centre les quantités de sodium sont de 6,14 et $5,37 \mathrm{~g} / \mathrm{kg}$.

$\mathrm{Au}$ contraire, en présence de pimaricine, la répartition du sodium dans le fromage est homogène ; à 19 jours la teneur de la croûte est de $4,11 \mathrm{~g} / \mathrm{kg}$ et de $4,75 \mathrm{~g} / \mathrm{kg}$ pour les 2 autres parties. 

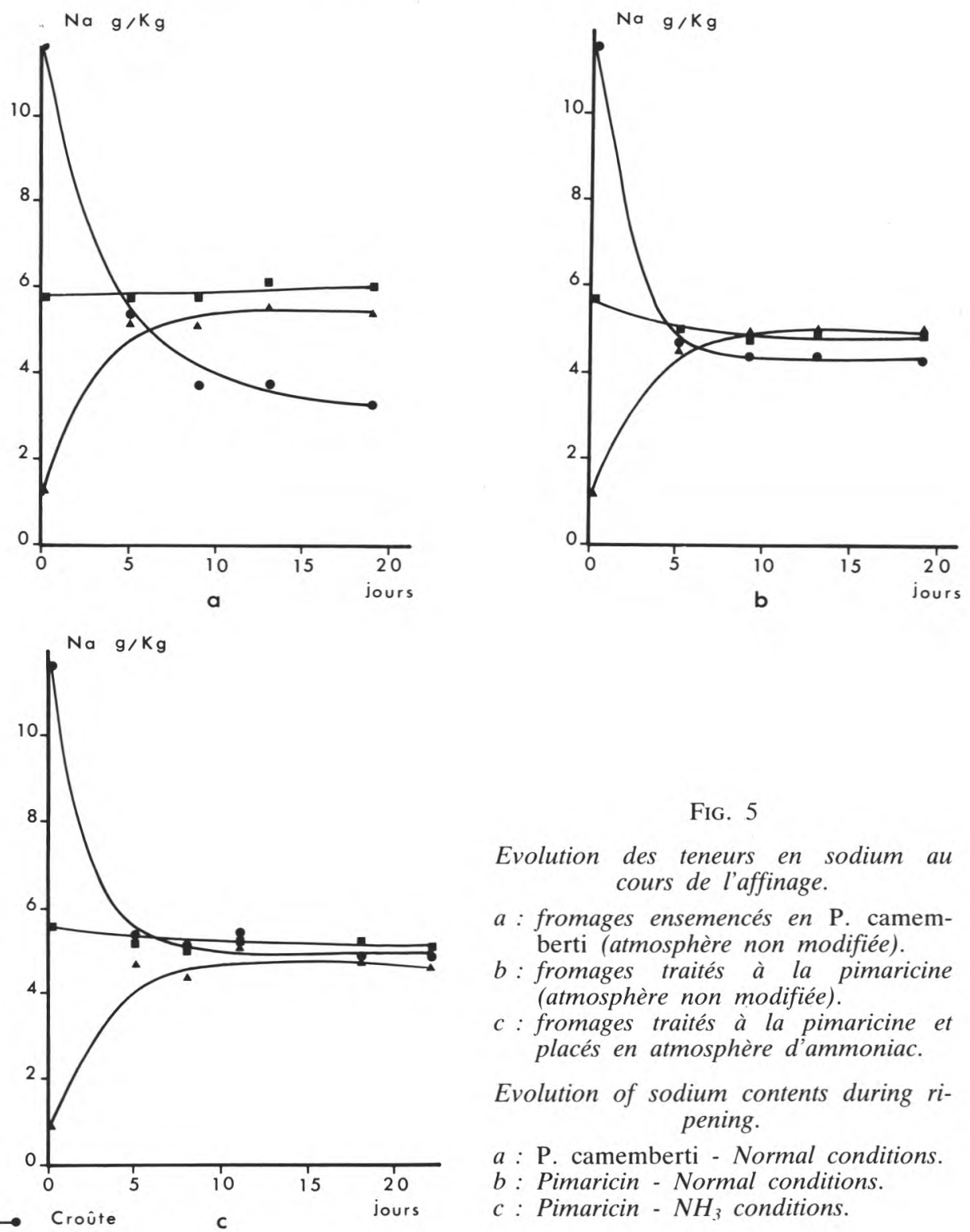

FIG. 5

Evolution des teneurs en sodium au cours de l'affinage.

$a$ : fromages ensemencés en $\mathrm{P}$. camemberti (atmosphère non modifiée).

$b$ : fromages traités à la pimaricine (atmosphère non modifiée).

$c$ : fromages traités à la pimaricine et placés en atmosphère d'ammoniac.

Evolution of sodium contents during ripening.

a : P. camemberti - Normal conditions.

$b$ : Pimaricin - Normal conditions.

c : Pimaricin - $\mathrm{NH}_{3}$ conditions.

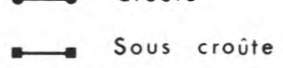

$\longrightarrow$ Centre

L'utilisation d'ammoniac et de pimaricine ne modifie pas de façon importante la répartition du sodium dans le fromage; ainsi à 22 jours les teneurs de la croûte, partie sous-croûte et du centre sont respectivement de 4,58, 4,98 et $4,40 \mathrm{~g} / \mathrm{kg}$. 


\section{Répartition du potassium}

Les fromages ensemencés en penicillium montrent dès 5 jours une augmentation de la teneur en potassium dans la croûte qui atteint un maximum à 9 jours et diminue ensuite constamment jusqu'à obtention d'un équilibre entre les 3 zones (fig. 6). Les teneurs de la croûte sont de $1,25 \mathrm{~g} / \mathrm{kg}$ à 5 jours, $3,37 \mathrm{~g} / \mathrm{kg}$ à 9 jours et $1,24 \mathrm{~g} / \mathrm{kg}$ à 19 jours. Durant la même période les teneurs de la partie sous-croûte et du centre sont de 0,99 et $1,03 \mathrm{~g} / \mathrm{kg}$ à 5 jours, 0,85 et $0,80 \mathrm{~g} / \mathrm{kg}$ à 9 jours et 1,26 et $1,10 \mathrm{~g} / \mathrm{kg}$ à 19 jours.
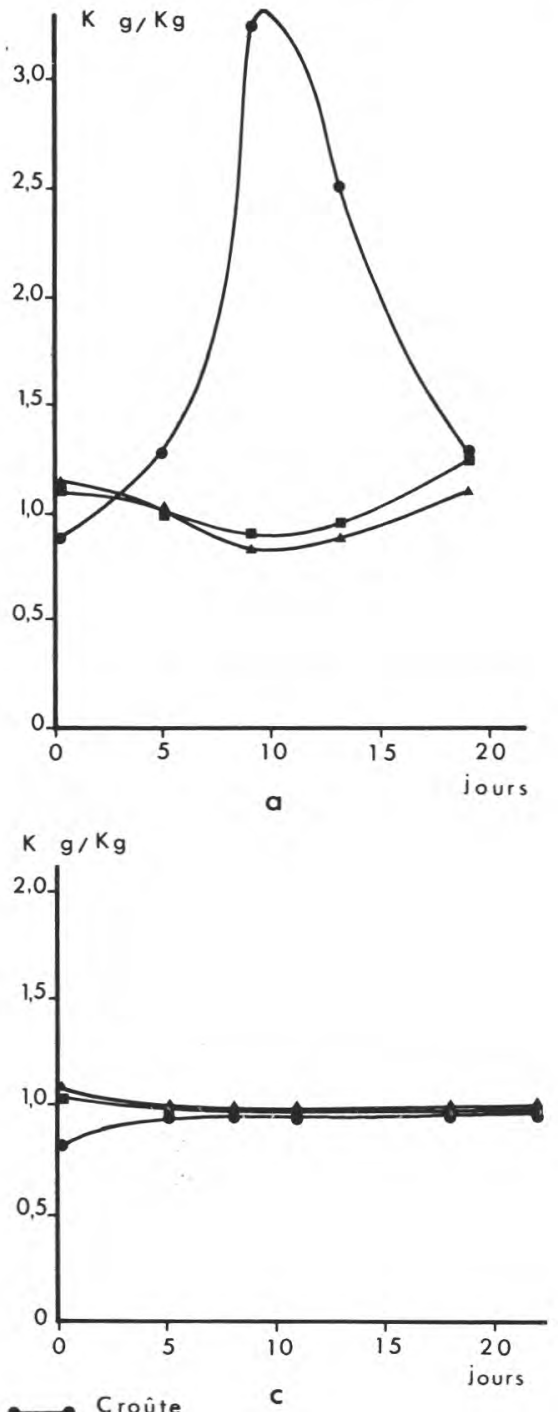

- Sous croûte

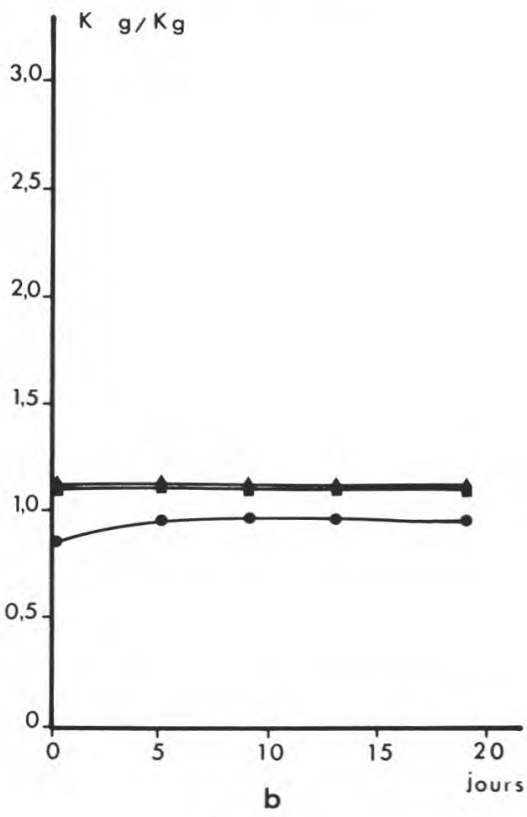

FIG. 6

Evolution des teneurs en potassium au cours de l'affinage.

$a$ : fromages ensemencés en $\mathrm{P}$. camemberti (atmosphère non modifiée).

$b$ : fromages traités à la pimaricine (atmosphère non modifiée).

$c$ : fromages traités à la pimaricine et placés en atmosphère d'ammoniac.

Evolution of potassium contents during ripening.

$a$ : P. camemberti - Normal conditions.

$b$ : Pimaricin - Normal conditions.

c: Pimaricin - $\mathrm{NH}_{3}$ conditions. 
En présence de pimaricine aucune variation n'est observée et à 19 jours les valeurs dans la croûte sont de $0,92 \mathrm{~g} / \mathrm{kg}$ et de $1,10 \mathrm{~g} / \mathrm{kg}$ dans les 2 autres parties du fromage.

Il en est de même en atmosphère d'ammoniac où aucune variation n'est à noter entre les 3 parties : $0,93 \mathrm{~g} / \mathrm{kg}$ dans la croûte, $1,02 \mathrm{~g} / \mathrm{kg}$ dans la partie sous-croûte et $0,95 \mathrm{~g} / \mathrm{kg}$ dans le centre à 22 jours.

\section{Répartition des oligo-éléments}

1. Répartition du zinc

A 10 jours la teneur en zinc de la croûte avec moisissure passe par un maximum pour diminuer ensuite (fig. 7). Ainsi la quantité de zinc dans la croûte est de $35,8 \mathrm{mg} / \mathrm{kg}$ à 5 jours, $89,6 \mathrm{mg} / \mathrm{kg}$ à 9 jours et seulement $57,6 \mathrm{mg} / \mathrm{kg}$ à 19 jours. Durant la même période les valeurs dans la partie souscroûte et le centre sont 25,3 et $24,6 \mathrm{mg} / \mathrm{kg}$ à 5 jours, 27,8 et $18,3 \mathrm{mg} / \mathrm{kg}$ à 9 jours, 28,5 et $20,4 \mathrm{mg} / \mathrm{kg}$ à 19 jours.
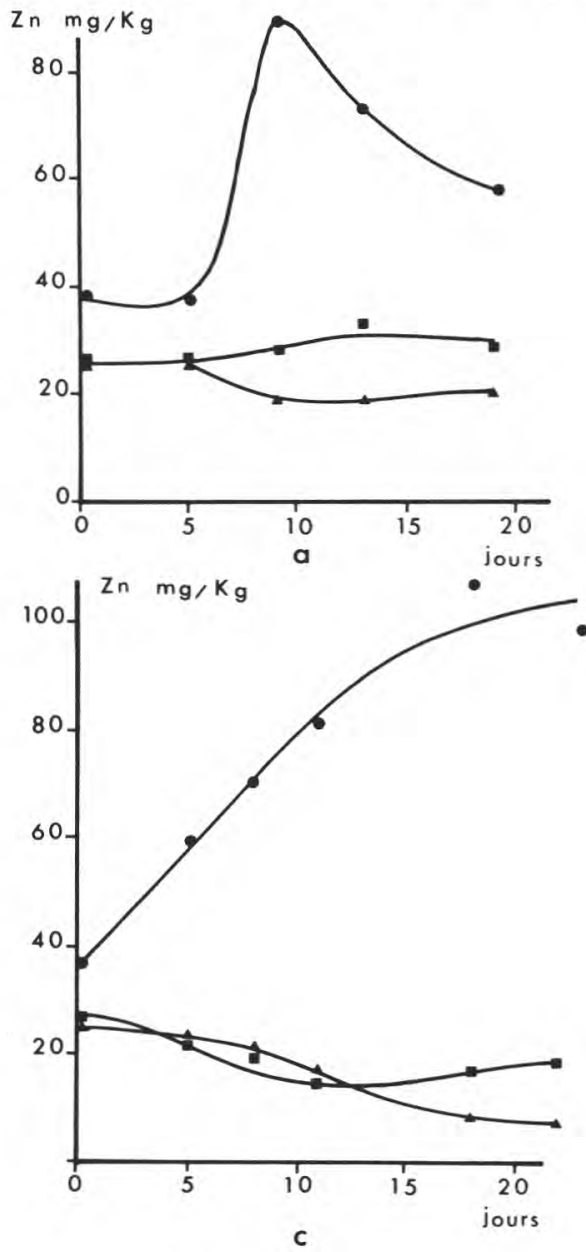

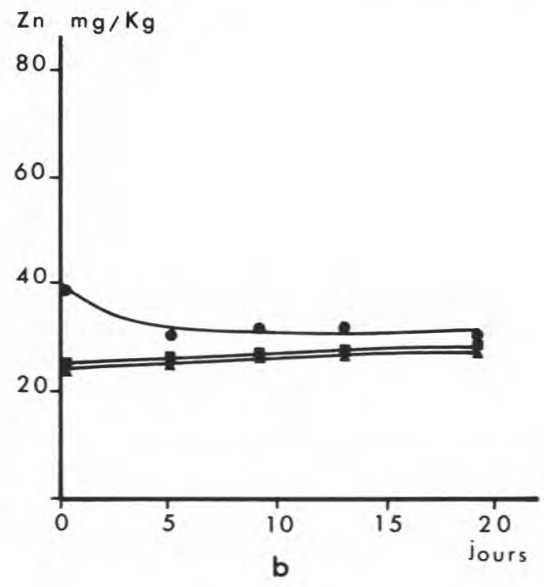

FIG. 7

Evolution des teneurs en zinc au cours de l'affinage.

$a$ : fromages ensemencés en P. camemberti (atmosphère non modifiée).

$b$ : fromages traités à la pimaricine (atmosphère non modifiée).

$c$ : fromages traités à la pimaricine et placés en atmosphère d'ammoniac.

Evolution of zinc contents during ripening.

$a$ : P. camemberti - Normal conditions.

$b$ : Pimaricin - Normal conditions.

c : Pimaricin - $\mathrm{NH}_{3}$ conditions.

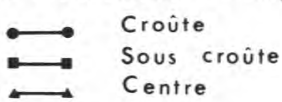


L'utilisation de pimaricine donne une répartition homogène durant la période d'essai ; à 19 jours, les valeurs en zinc de la croûte, sous-croûte et du centre sont : $31,2,28,7$ et $26,5 \mathrm{mg} / \mathrm{kg}$.

En atmosphère ammoniacale la teneur en zinc de la croûte augmente de façon continue dès 5 jours et celle de la sous-croûte et du centre diminue constamment. Les teneurs en zinc de la croûte, partie sous-croûte et du centre sont de $59,8,21,5$ et $23,2 \mathrm{mg} / \mathrm{kg}$ à 5 jours, de $108,4,17,6$ et $8,87 \mathrm{mg} / \mathrm{kg}$ à 18 jours.

\section{Répartition du fer}

En atmosphère non modifiée les fromages ensemencés en penicillium ont une teneur en fer de leur croûte qui augmente dès le $5^{\mathrm{c}}$ jour alors que dans les 2 autres parties elle diminue (fig. 8). Les quantités de fer observées dans la croûte, partie sous-croûte et le centre s'élèvent à $1,48,0,75$ et $0,63 \mathrm{mg} / \mathrm{kg}$ à 5 jours ; à 19 jours elles sont de $2,67 \mathrm{mg} / \mathrm{kg}$ dans la croûte et $0,42 \mathrm{mg} / \mathrm{kg}$ dans les 2 autres parties du fromage.
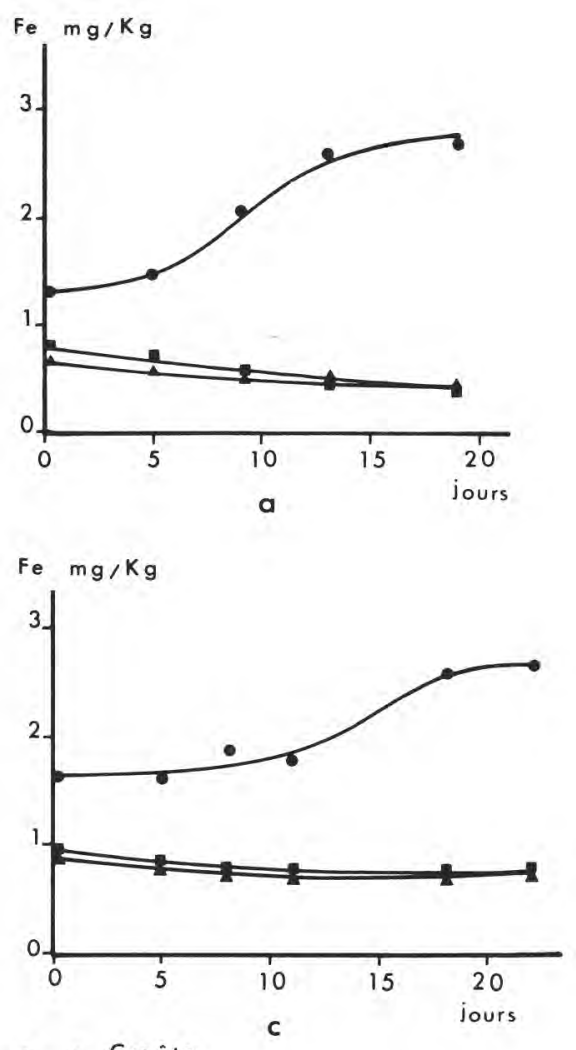

$\longrightarrow$ Croûte

$\longrightarrow$ Sous croûte

$\longleftrightarrow$ Centre

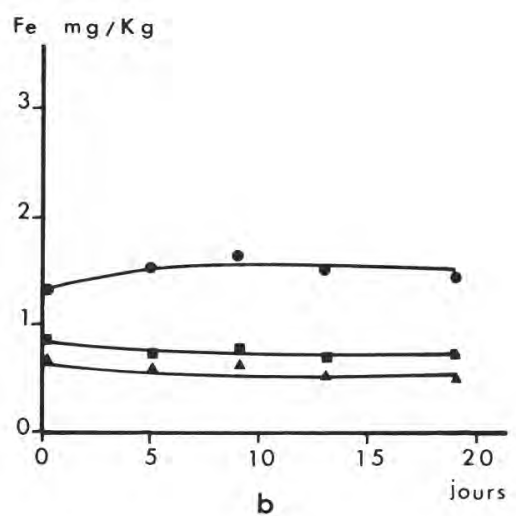

FIG. 8

Evolution des teneurs en fer au cours de l'affinage.

$a$ : fromages ensemencés en P. camemberti (atmosphère non modifiée).

$b$ : fromages traités à la pimaricine (atmosphère non modifiée).

$c$ : fromages traités à la pimaricine et placés en atmosphère d'ammoniac.

Evolution of iron contents during ripening.

a : P. camemberti - Normal conditions.

b : Pimaricin - Normal conditions.

c: Pimaricin - $\mathrm{NH}_{3}$ conditions. 
L'utilisation de pimaricine met en évidence qu'il n'y a pas d'évolution significative dans la répartition du fer entre les 3 zones du fromage.

En atmosphère ammoniacale on note une faible augmentation de la teneur en fer de la croûte, $1,64 \mathrm{mg} / \mathrm{kg}$ à 5 jours et $2,58 \mathrm{mg} / \mathrm{kg}$ à 22 jours ; dans la partie sous-croûte et le centre les valeurs sont respectivement 0,80 et $0,92 \mathrm{mg} /$ $\mathrm{kg}$ et $0,73 \mathrm{mg} / \mathrm{kg}$ pour chacune des 2 parties et pour la même période.

3. Répartition du cuivre

Dans les 3 cas étudiés la répartition du cuivre dans le fromage est homogène entre les 3 zones et les teneurs se situent aux environs de $0,50 \mathrm{mg} /$ $\mathrm{kg}$ (fig. 9).
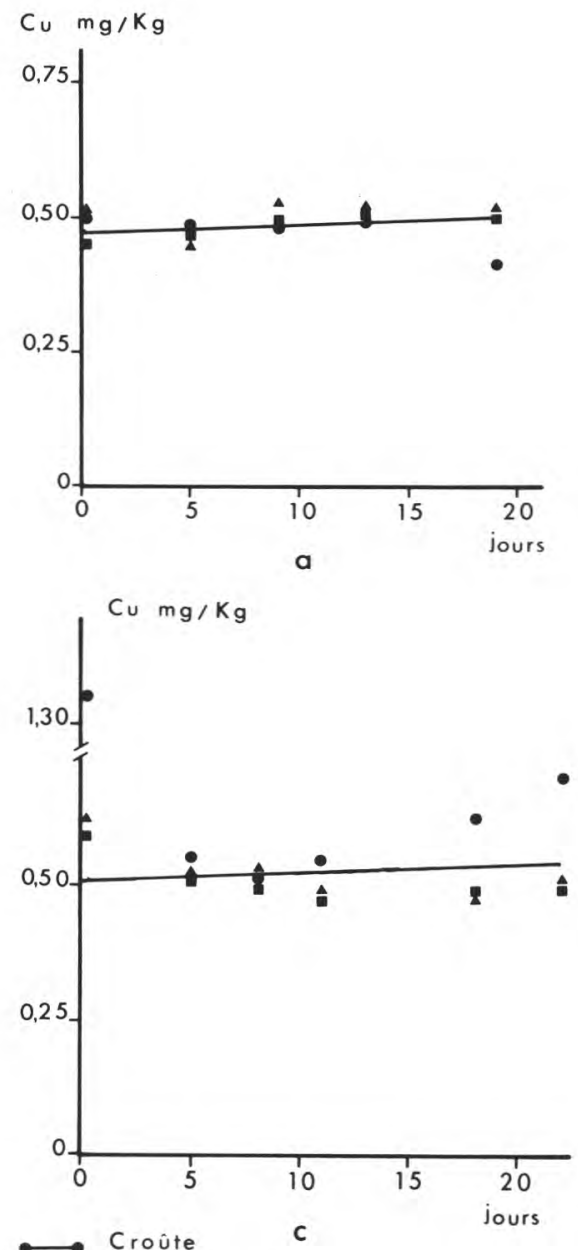

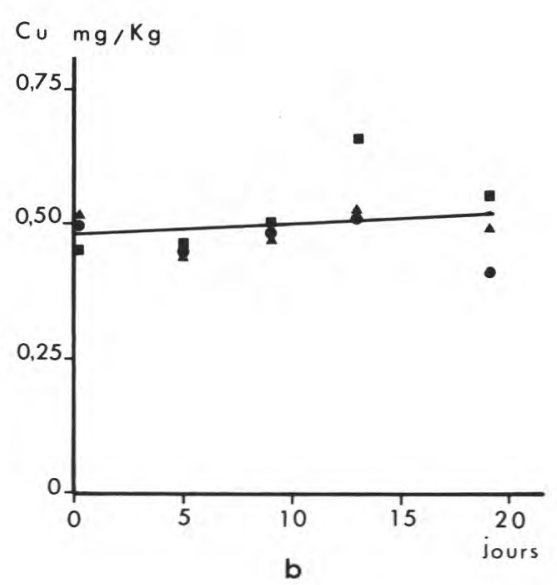

FIG. 9

Evolution des teneurs en cuivre au cours de l'affinage.

$a$ : fromages ensemencés en P. camemberti (atmosphère non modifiée).

$b$ : fromages traités à la pimaricine (atmosphère non modifiée).

$c$ : fromages traités à la pimaricine et placés en atmosphère d'ammoniac.

Evolution of copper contents during ripening.

$a$ : P. camemberti - Normal conditions.

$b$ : Pimaricin - Normal conditions.

$c$ : Pimaricin $-\mathrm{NH}_{3}$ conditions.

$\longrightarrow$ Sous croûte 


\section{Discussion}

Les résultats obtenus confirment ceux d'une étude précédente (LE GraET et al., 1983) et mettent en évidence le rôle du penicillium et du $\mathrm{pH}$ sur la migration minérale et ses incidences sur le transfert d'eau dans le fromage.

L'évolution du magnésium au cours de l'affinage est semblable à celle du calcium. Néanmoins, en fin d'affinage le rapport :

\section{Teneur de l'élément dans la croûte \\ Teneur de l'élément dans le centre}

est de 7 environ pour le magnésium et de 18 pour le calcium. Il en résulte que le magnésium migre 2,5 fois moins que le calcium, et, aurait donc moins d'affinité pour l'ion phosphate. L'insolubilité des phosphates de calcium et de magnésium étant identique, on peut supposer que seule leur différence d'encombrement stérique conduit à une affinité préférentielle pour le calcium.

Le potassium nécessaire au développement du penicillium présente la particularité de migrer durant la phase de croissance de ce dernier avec une teneur maximum de $3,37 \mathrm{~g} / \mathrm{kg}$ dans la croûte à 9 jours. La lyse partielle des cellules du penicillium ou une modification de la perméabilité de leur membrane, à partir de 9 jours libérerait du potassium qui migrerait cette fois en sens inverse. Le prélèvement de potassium par le penicillium appauvrit la teneur de la phase soluble et l'équilibre se rétablit par déplacement de l'élément de l'intérieur vers l'extérieur du fromage. Par contre, en fin d'affinage la répartition en potassium dans le fromage est homogène malgré un ES différent de chacune des parties; la croûte ayant en plus une composition physico-chimique très différente. En présence de pimaricine, la teneur moindre de la croûte, peut être liée à un ES plus élevé que dans le reste du fromage, donc à une quantité d'eau solvante moindre.

L'ion sodium se comporte différemment de l'ion potassium. Au cours de l'affinage, il ne présente dans la croûte aucun maximum susceptible de correspondre à la phase de croissance du penicillium, mais au contraire une diminution constante. Il ne semble donc pas y avoir de besoins spécifiques marqués en cet élément par le penicillium à moins qu'il ne soit largement excédentaire. A 9 jours, les teneurs en potassium et en sodium de la croûte sont sensiblement identiques et se situent aux environs de $3,5 \mathrm{~g} / \mathrm{kg}$ mais, cette valeur représente respectivement 0,09 et 0,15 mole ; ces chiffres renforcent l'idée que le sodium peut être en excès. Sa faible teneur dans la croûte par rapport aux autres parties du fromage peut également s'expliquer par une forte teneur en eau liée du penicillium, eau qui de ce fait ne serait pas disponible. La partie externe du fromage plus pauvre en sodium que la partie interne a déjà été observée par HuREL et Mocquot (1947) et appelée «inversion des salures ».

Dans les fromages ayant reçu une pulvérisation de pimaricine la teneur en sodium est en rapport inverse de la teneur en ES; il en découle une phase soluble moins importante donc une quantité de sodium moindre.

La migration du zinc se fait probablement soit sous l'influence du gradient de $\mathrm{pH}$, soit pour satisfaire les besoins de la flore de surface. Il pourrait donc 
d'une part précipiter sous forme d'hydroxyde et/ou de phosphates en l'absence de chélatant tel que le citrate, et d'autre part être complexé par des macromolécules cellulaires ou intervenir comme cofacteur de systèmes enzymatiques dont notamment la métalloprotéase (GRIPON et al., 1980). Ces macromolécules libérées progressivement dans la pâte, mais ayant une faible diffusion (LENOIR, 1970 ; Noomen, 1983) provoqueraient une diminution de la teneur de la croûte en cet élément au seul profit de la partie sous-croûte. Dans la partie centrale, la faible augmentation de la teneur en zinc après 9 jours peut être liée à l'élévation de la teneur en ES; il serait concentré en même temps et dans le même rapport que les caséines. Le zinc est en effet fortement fixé aux caséines (Brule et Fauquant, 1982); il en est de même à $\mathrm{J} 0$ où les teneurs de la croûte sont plus élevées en ES, donc en zinc, que les 2 autres parties du fromage.

Pour les mêmes raisons que celles énoncées pour le zinc, le fer se trouve initialement en quantité plus importante dans la croûte que dans le reste du fromage; néanmoins, une partie de la teneur en fer peut être liée aux contaminations toujours possibles, notamment au cours de la fabrication. A partir de 5 jours, le fer migre vers la croûte et précipite à la surface du fromage sous forme d'hydroxyde et/ou de phosphates insolubles sous l'influence du gradient de $\mathrm{pH}$. Seul le gradient de $\mathrm{pH}$ semble prépondérant dans la migration du fer.

Le cuivre est très fortement fixé aux protéines (Brulé et FAUQuANT, 1982 ; Baumy, 1987) par des liaisons différentes de celles des autres éléments et ne migre pas quel que soit le $\mathrm{pH}$; en effet, les liaisons de coordination qui interviennent dans la formation de ces complexes ne sont pas sous la dépendance du $\mathrm{pH}$. Les teneurs de la croûte sont plus élevées que celles du reste du fromage pour les mêmes raisons que celles évoquées pour le zinc et le fer.

Il ressort de cette étude que tous les éléments ne migrent ou ne se répartissent pas dans les mêmes rapports, même pour 2 éléments aussi proches que calcium et magnésium ou sodium et potassium. Ces minéraux ne précipitent pas à la surface du fromage sous la même forme : essentiellement des phosphates pour le calcium et le magnésium et probablement d'hydroxydes et de phosphates pour le zinc et le fer. Les modifications de la composition minérale du fromage par appauvrissement de la partie interne en certains éléments au profit de la croûte jouent peut-être un rôle au cours de l'affinage notamment au niveau du développement de la flore. Cependant, cet aspect de nutrition minérale des microorganismes présente de nombreuses lacunes au niveau qualitatif et quantitatif. Par ailleurs, les teneurs en minéraux disponibles pour la flore du fromage, c'est-à-dire vraisemblablement ceux contenus dans la phase soluble, demeurent encore peu connues. 


\section{Références bibliographiques}

Baumy J.J., 1987. Résultats non publiés.

Brulé G., Fauquant J., 1982. Interaction des protéines du lait et des oligo-éléments. Lait, 62, 323-331.

Gripon J.C., Auberger B., Lenoir J., 1980. Metalloproteases from Penicillium caseicolum and Penicillium roqueforti. Comparison of specificity and chemical characterization. Int. J. Biochem., 12, 451-455.

Hofi A.A., Rifaat J.D., Salam M.H.A., Mahran G.A., 1970. Utilisation des oligo-éléments pour accélérer l'affinage des fromages à pâte dure. XVIII ${ }^{\mathrm{e}}$ Congrès International de Laiterie.

Hurel C., Mocquot G., 1947. Etude comparée des fromages de Livarot et de Pont-l'Evêque. Lait, 27, 131-141.

Lenolr J., 1970. L'activité protéasique dans les fromages à pâte molle de type Camembert. Rev. Lait. Fr., (275), 231-243.

Lenoir J., Glenza A., Bergère J.L., Cerf O., Choisy C., Desmazeaud M., Hermier J., 1973. Les facteurs de production du système protéolytique de Penicillium caseicolum. Lait, 53, 246279.

Le Graet Y., Lepienne A., Brulé G., Ducruet P., 1983. Migration du calcium et des phosphates inorganiques dans les fromages à pâte molle de type Camembert au cours de l'affinage. Lait, 63, 317-332.

le Graet Y., Brulé G., Maubois J.L., Oeuvrard G., 1986. Répartition et évolution des éléments minéraux au cours de l'affinage des fromages à pâte cuite type Beaufort. Lait, 66, 391-404.

Noomen A., 1983. The role of surface flora in the softening of cheeses with a low initial $\mathrm{pH}$. Neth. Milk Dairy J., 37, 229-232.

SAAKYAN R.V., 1982. Use of trace elements in Swiss cheese manufacture. Molochnaya Promyshlennost, 3, 27-29 (Dairy Sci. Abstr., 45, 104).

SAAKYAN R.V., 1982. Effect of trace elements on the ripining and quality of Swiss and Sovetskii Cheeses. Izvestiya Vysohikh Uchelnykh Zavedenu Pishchevaya Teckhnologiya, 5, 57-60 (Dairy Sci. Abstr., 45, 812). 